\title{
Differential attentional responding in caesarean versus vaginally delivered infants
}

\author{
Scott A. Adler ${ }^{1,2}$ • Audrey M. B. Wong-Kee-You ${ }^{1}$
}

Published online: 11 August 2015

(C) The Psychonomic Society, Inc. 2015

\begin{abstract}
Little is known about the role that the birth experience plays in brain and cognitive development. Recent research has suggested that birth experience influences the development of the somatosensory cortex, an area involved in spatial attention to sensory information. In this study, we explored whether differences in spatial attention would occur in infants who had different birth experiences, as occurs for caesarean versus vaginal delivery. Three-month-old infants performed either a spatial cueing task or a visual expectation task. We showed that caesarean-delivered infants' stimulus-driven, reflexive attention was slowed relative to vaginally delivered infants', whereas their cognitively driven, voluntary attention was unaffected. Thus, types of birth experience influence at least one form of infants' attention, and possibly any cognitive process that relies on spatial attention. This study also suggests that birth experience influences the initial state of brain functioning and, consequently, should be considered in our understanding of brain development.
\end{abstract}

Keywords Infants · Development · Attention · Bottom-up · Top-down $\cdot$ Vaginal birth $\cdot$ Caesarean section $\cdot$ Eye movements

Many studies have documented the critical role that experience plays in the development of brain structures and cognitive behaviors (Diamond \& Amso, 2008; Greenough \& Black,

Scott A. Adler

adler@yorku.ca

1 Department of Psychology and Centre for Vision Research, York University, Toronto, Ontario, Canada

2 York University, 5030 TEL, 4700 Keele Street, Toronto, Ontario M3J 1P3, Canada
2013). Experience is known, for example, to alter the formation, maintenance, and pruning of synapses through the mechanisms of neural plasticity during development (Aoki \& Erisir, 2013; Maurer, Ellemberg, \& Lewis, 2006). Experience with multiple versus a single language early in development has been shown to have a lasting impact on cognitive functioning, and likely on the supporting neural substrate (Mohades et al., 2012). Experience with method of feeding, breast-feeding or formula, has been demonstrated to influence cognitive development (Quigley et al., 2012). As Greenough and Black (2013) described, experience has a dramatic impact on synaptic structure, through either processes that are inherent to the system to apprehend information that is impinging on them or processes that serve to modulate synaptic formation that are unique to each individual's set of distinct exposures to environmental events. Through these processes, experiences starting from early in development are intimately related to synaptic structure and, hence, to brain and cognitive status throughout development. Yet the role of the earliest experience, that of birth itself, on any aspect of cognitive development has never been investigated.

Various studies over a number of decades have demonstrated the role very early birth factors have on aspects of cognitive development, including attentional responding. These earlier studies have assessed, for example, the cumulative role of obstetric factors, including an infant's gestational age, the mother's age, and birth weight, and found that they are related to attentional performance (i.e., habituation and orientation) on the Brazelton Neonatal Behavioural Assessment Scale (Sepkoski, Garcia-Coll, \& Lester, 1982). Studies have also shown, for example, that receiving an epidural during a vaginal birth was related to poorer attentional orientation in 1-month-old infants, though other birth factors, including length of labor, had no impact (Sepkoski, Lester, Ostheimer, \& Brazelton, 1992). Additionally, numerous studies have 
investigated the attentional effects of being born preterm relative to full-term (e.g., Shum, Neulinger, O'Callaghan, \& Mohay, 2008; van de Weijer-Bergsma, Wijnroks, \& Jongmans, 2008). None of these studies, or any others, however, have investigated the role that the birth experience itself plays in cognitive development in general, or attentional development in particular.

Birth experience can take different forms, and the relative effects of the different forms - for example, vaginal versus caesarean-section delivery - on cognitive and brain development has never been explored. Recent studies on the potential health effects of being delivered by caesarean section have indicated that caesarean section increases a child's risk of contracting diabetes, developing allergies, and becoming obese (Cardwell et al., 2008; Flemming, Woolcott, Allen, Veugelers, \& Kuhle, 2013; Renz-Polster et al., 2005). None of these studies detailing potential health risks for children born by caesarean section, however, have examined whether any brain or cognitive developmental consequences emerge from the caesarean-section birth experience, relative to the birth experience of being delivered vaginally. In the present study, therefore, we explored one of 3-month-old infants' cognitive mechanisms, namely visual attention and related eye movements, in two distinct tasks as a function of whether they had experienced a vaginal or a caesarean-section delivery.

Recently, a study by Toda et al. (2013) demonstrated that birth experience plays a role in the brain development of rat pups and that variant birth experiences resulted in brain development differences. In particular, the study indicated that birth experience affected the postnatal development of the somatosensory cortex by mediating changes in the level of serotonin in the brain. When rats were artificially induced to prematurely give birth, barrel formation (equivalent to humans' sensory maps) in the pups' somatosensory cortex was accelerated. This acceleration in somatosensory cortex differentiation postnatally was attributed to a reduction in serotonin initiated by birth (Toda et al., 2013). Differences, though not significant, were also observed when comparing vaginally delivered pups to pups delivered by caesarean section, in that barrel formation in somatosensory cortex was slowed in the caesarean-sectiondelivered pups. Changes in birth experience, therefore, such as being born premature, or even being delivered by caesarean section, likely affect early differentiation of the somatosensory cortex.

A function of the somatosensory cortex in humans has been shown to be the moderation of attentional allocation and linked eye movements to the spatial characteristics of sensory events (Balslev, Odoj, \& Karnath, 2013; Jones et al., 2010). If the somatosensory cortex is involved in spatial attention and if its development in humans is affected by birth experience, as it is in rats (Toda et al., 2013), then infants' spatial attention might be disrupted by the lack of a natural birth experience, as occurs with a caesarean-section delivery. Consequently, we tested two independent groups of 3-month-old infants, each performing a distinct spatial attention task (see Fig. 2 below). One group performed a Posner-like spatial-cueing task in which a peripheral cue was presented that indicated the subsequent location of the target stimulus for the infants' saccadic eye movement. Extensive research has demonstrated that this spatial-cueing task activates bottom-up, stimulus-driven spatial attention (for a review, see Theeuwes \& Belopolsky, 2010). That is, the spatial cue elicits bottom-up attention to shift to its location prior to presentation of a target at that location (Klein \& Lawrence, 2012). As a consequence, since eye movements are linked to spatial attention (Adler, Bala, \& Krauzlis, 2002; Hoffman \& Subramaniam, 1995; Kowler, Anderson, Dosher, \& Blaser, 1995; Van der Stigchel \& Theeuwes, 2007), eye movement responses are facilitated to a target presented at the cued location relative to when the target location has not been cued.

The second group of infants performed a visual expectation task in which the stimuli predictably alternately appeared on the left and right sides of a monitor, engendering an increase in the level of saccadic eye movements in anticipation of the future appearance of the forthcoming stimulus. Because the anticipatory eye movements are guided by cognitive expectations of which stimulus will appear where and when (Adler \& Haith, 2003; Adler, Haith, Arehart, \& Lanthier, 2008; Haith, Hazan, \& Goodman, 1988), this task primarily activates topdown, cognitively driven spatial attention.

A number of models of visual attention development have postulated that the neural and attentional mechanisms necessary for reactive, bottom-up attentional selection are functional in the first few months of life (Atkinson, 1984, 2000; Braddick \& Atkinson, 2011; Johnson, 1995, 2002). These same models further postulate that the mechanisms necessary for top-down guidance of attentional allocation do not begin to show functionality until after 3 months of age, and likely are not fully functional until at least 6 months of age. Thus, infants 3-4 months of age would rely more on bottom-up mechanisms for allocating attentional resources than on less mature top-down attentional mechanisms. As a consequence, bottomup attentional allocation and the resulting responding should be more susceptible to potential disruption. Since attentional responding in the present study was in the overt form of eye movements, and it is well accepted that a tight linkage exists between attentional allocation and the initiation or orienting of eye movements (e.g., Adler et al., 2002; Hoffman \& Subramaniam, 1995; Kowler et al., 1995; Van der Stigchel \& Theeuwes, 2007), such that the eyes cannot go where attention is not, any disruption of attentional mechanisms, therefore, will be manifested in subsequent eye movements.

We therefore predicted that, by virtue of the hypothesized effect that birth experience has on the somatosensory cortex (Toda et al., 2013) and its role in bottom-up, stimulus-driven spatial attention (Balslev et al., 2013; Jones et al., 2010), 3- 
month-old infants' bottom-up attention would be affected by birth experience. In a spatial-cueing task in which a brief peripheral cue presented at the spatial location of a subsequent target stimulus is known to facilitate bottom-up attention (Theeuwes \& Belopolsky, 2010), therefore, we predicted that infants delivered by caesarean section would exhibit poorer attentional responding, in the form of reactive saccadic eye movements, relative to vaginally delivered infants. In a visual expectation task, however, which requires the formation of top-down cognitive expectations for a predictable sequence of pictures (Adler \& Haith, 2003; Adler et al., 2008; Haith et al., 1988), we predicted that top-down attention, as manifested by anticipatory eye movements to the spatial locations of forthcoming stimuli, would be unaffected by birth experience.

\section{Experiment 1: Spatial-cueing task}

\section{Method}

Participants Twenty-four infants (15 males, 9 females), who ranged in age from 98 to 125 days old $(M=104, S D=11)$, participated in this experiment. They were pseudorandomly assigned to one of three independent experimental conditions $(n=8)$, with the criterion that equal numbers $(n=4)$ of caesarean-section and vaginally delivered infants were in each condition. The infants in the sample were Caucasian $(n=14)$, Hispanic $(n=2)$, Asian $(n=3)$, East Asian $(n=2)$, African $(n=2)$, and other $(n=1)$, and were primarily of middle to upper socioeconomic status (SES). The infants (12 delivered via caesarean section, 12 delivered vaginally) were all born full-term, within \pm 2 weeks of the self-reported due dates, and appeared to be in good health, with no apparent visual or neurological abnormalities. The data from 10 additional infants who participated were excluded from the study because of fussiness $(n=5)$, equipment or software failure $(n=1)$, or inattentiveness (i.e., infants were disinterested or looked away from the visual field on more than $50 \%$ of trials; $n=4$ ).

Stimuli and apparatus During both experimental tasks, the babies were laid supine in a specialized crib and viewed stimuli on an LCD monitor, mounted $48 \mathrm{~cm}$ above. Between the infant and the monitor was a $30 \times 30 \mathrm{~cm}$ infrared-reflecting, visible-transmitting mirror that provided the infant with an unobstructed view of the stimuli on the monitor. A remote pan-tilt infrared eyetracking camera (Model 504, Applied Science Laboratories [www.a-s-1.com], Bedford, MA) was also placed overhead (see Fig. 1). Black felt curtains were hung around the crib to limit light entry and reduce distraction.

Using bright-pupil technology, the pan-tilt eyetracker recorded the infants' eye movements via reflection in the infrared mirror at a temporal resolution of $60 \mathrm{~Hz}$. Diodes on the

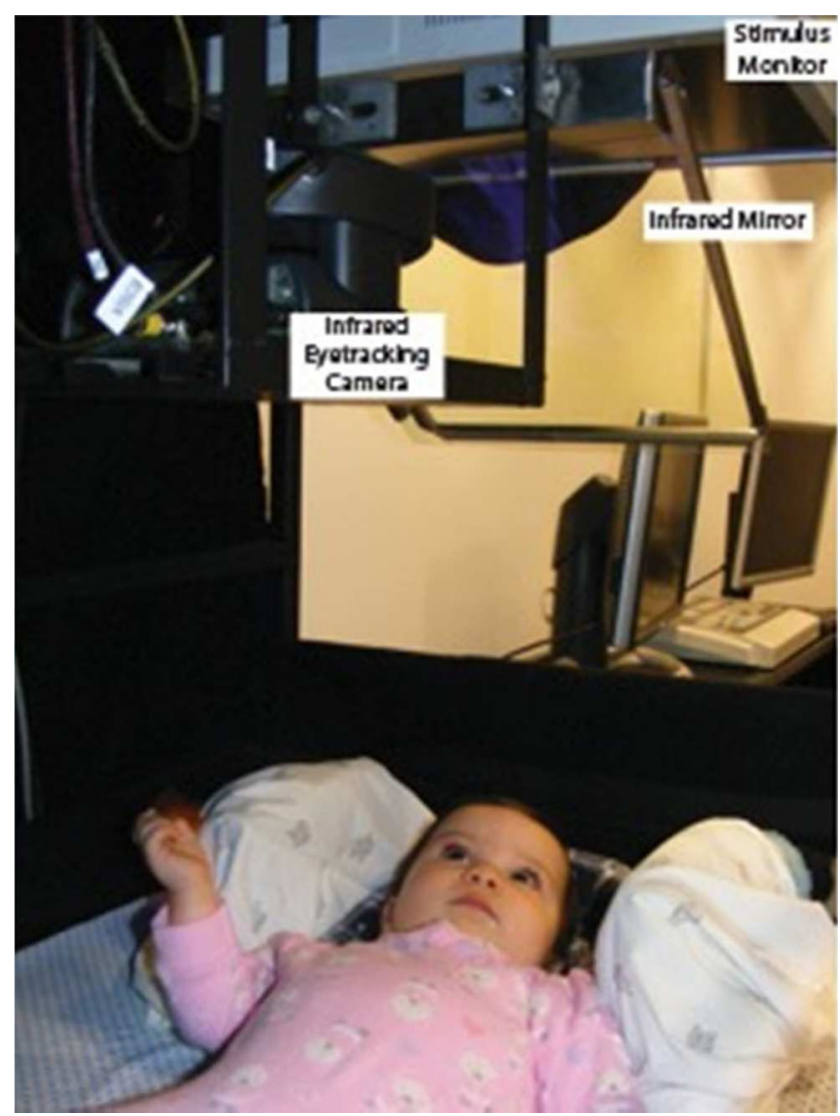

Fig. 1 Image of the specialized crib used with infants, showing the monitor on which stimuli were presented, the Model 504 infrared eyetracking camera, and the infrared mirror

camera emitted infrared light that reflected off the infrared mirror onto and back off the infant's retina through the pupil, to produce a backlit white pupil. The infrared light also produced a point reflection on the corneal surface of the eye. Through proprietary software (Applied Science Laboratories), the eye fixation position was calculated from the relation between the corneal reflection and the centroid of the backlit pupil. The eyetracker was initially calibrated by having the infant look at a stimulus (concentric squares) that was presented at known locations on the LCD screen. This calibration was done in order to equate the recorded eyetracker values of eye location to known locations on the screen. All subsequently recorded eyetracker values were filtered through the calibration file to produce measures of eye position data.

Two Dell computers were used during the experimental session. One computer generated and presented the stimuli using the program DirectRT (Empirisoft Inc., New York, NY; www.empirisoft.com/DirectRT.aspx), whereas the other computer controlled the eyetracker camera and collected the eye movement data. The experimenter viewed the infant's eye movements and stimulus presentation on the data collection computer as a picture-in-picture video, via video capture software. The stimulus-generating computer sent a unique, timestamped numerical code through a parallel port to the data- 
collecting computer, indicating the onset of a trial and the type of trial (e.g., cue with target and distractor in the spatial-cueing task, or an invariant color stimulus on either the right or the left side of the screen in the visual expectation task). Synchronization of the unique code with the eye movement data in the data file allowed coordination of the eye movement sequences with specific stimuli and their onsets.

Procedure Infants viewed stimuli in a sequence based on the Posner (1980) cueing paradigm. In the cued conditions, a trial began with the presentation of a fixation hexagon for $1,150 \mathrm{~ms}$ (see Fig. 2A). During the last $150 \mathrm{~ms}$ of the presentation of the fixation hexagon, a spatial cue (white triangle with a black outline) was presented at the location where the target would subsequently appear. Immediately after the presentation of the fixation and the cue, an interstimulus interval (ISI) of 2,500 ms was presented, during which the monitor was blank. A previous study by Gilmore and Johnson (1995) had shown that older infants exhibit eye movement facilitation to a cued target in the presence of a distractor with delays up to 3,000 ms. Furthermore, as was indicated by Johnson (2002), attentional shifts to the stimulus opposite the cued target due to inhibition of return is evident in infants 4 months of age and older, but likely not in younger infants. Considering that 3-month-olds are more likely to demonstrate sticky fixation than are older infants (Hunnius \& Geuze, 2004), we chose this ISI so that infants would have sufficient time to disengage attention from fixation and still show facilitation to a cued target. Following the ISI, the target stimuli were presented, appearing on either the right or the left of the screen (target-only condition) or on both sides (target + distractor condition). The target stimuli were red or green Xs presented at a visual angle of $5.5^{\circ}$ from the visual center of the screen. For the uncued conditions, the sequence of stimulus presentation was similar, but no spatial cue was presented and only the target-only condition was run. In all conditions, the latencies of the infants' reactive eye movements to the target were measured.

\section{A Spatial Cueing Task}

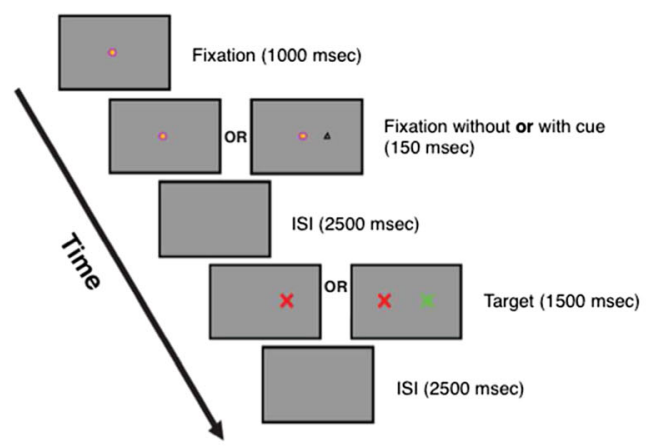

Fig. 2 Schematics of the two spatial attention tasks used with the infants. (A) In the spatial cueing task (Exp. 1), infants either were briefly presented with a cue that indicated the subsequent location of the target or were
Data reduction and analysis The raw digital data recorded by the eyetracker were imported into a MATLAB toolbox called ILAB for analysis (Gitelman, 2002). ILAB allows for the analysis of eye movements, by parsing out and individually displaying the horizontal and vertical components of the eye movement data on a trial-by trial basis. The scan path of the eye for each trial was also displayed by ILAB, thereby allowing for the analysis of the nature of the eye movements (timing, direction, and distance) relative to the stimuli. With the use of ILAB, a scorer identified which of infants' eye movements were anticipatory or reactive in timing.

In order for an eye movement to be included in the final data sample, it needed to meet a set of criteria. First, only the data of infants who attended (i.e., looked at the stimuli) on a minimum of $50 \%$ of the experimental trials were included. Second, infants were required to be fixating the central stimulus during cue presentation and before onset of the target display. The purpose of requiring the infants to remain fixated was to allow for an assessment of the scan path of each eye movement from a single landmark that was a fixed distance from the target and, thus, to eliminate bias from producing eye movements that originated from random locations on the screen. Because infants cannot be told to remain fixated and can freely move their eyes at any time, this criterion ensured that the initial conditions for assessing infants' performance were comparable across infants, conditions, and trials. Third, eye movements were considered to be anticipatory if they occurred after the offset of the previous stimulus and within the first $167 \mathrm{~ms}$ after the onset of the next stimulus. This latency value was designated as the anticipatory cutoff because previous studies had determined that 3-month-old infants cannot make reactive eye movements to the onset of a stimulus faster than $167 \mathrm{~ms}$ (Adler \& Haith, 2003; Canfield, Smith, Brezsnyak, \& Snow, 1997). If an eye movement occurred in the period from $167 \mathrm{~ms}$ after onset of the stimulus to $167 \mathrm{~ms}$ after stimulus offset, it was classified as a reactive eye movement. Finally, the eye movement to a stimulus had to

\section{B Visual Expectation Task}

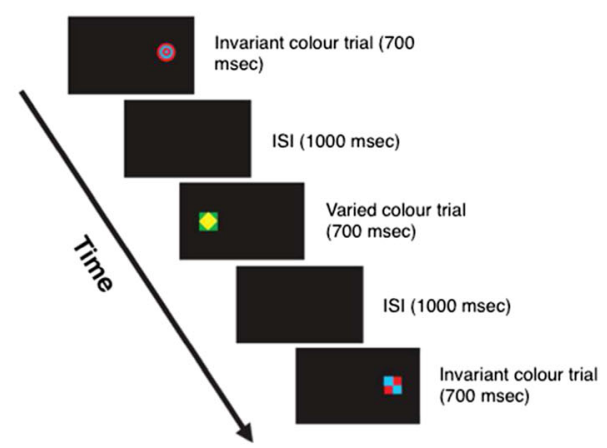

presented with no cue. (B) In the visual expectation task (Exp. 2), infants were presented with geometric colored patterns that predictably alternated between the left and right sides of the screen 
trace a path that was more than $50 \%$ of the distance to the intended stimulus from that eye movement's starting location. This was assessed through the infant's scan path in conjunction with the stimulus location. The $50 \%$ criterion has been used in previous studies based on infants' eye movements (Adler \& Haith, 2003; Adler \& Orprecio, 2006) and is typically taken as an indication that the eye movement is intentional and not random.

Due to the fact that one cannot require an infant to look on a given trial, the possibility existed that any given infant might not provide useful data in a particular condition. In order to account for potential missing data points and to increase the power of our statistical tests, the individual trial data from all the infants were pooled for each Experimental $\times$ Birth Experience condition, and analyses were based on the pooled data. This is consistent with a number of adult and infant studies that have used saccade latencies as the dependent measure (e.g., Adler et al., 2002; Adler \& Gallego, 2014). On the basis of previous infant eye movement studies, to obtain a power $(1-\beta)$ equal to .90 , a minimum of 21 observations per condition were required. With the pooling of the data, the smallest number of observations in any condition was 79 , and the most was $106(M=95.0, S D=10.1)$.

\section{Results}

In typical spatial-cueing tasks, evidence of the role of bottomup spatial attention in performance is provided by a comparison of responding to a target presented at a cued location relative to responding to a target presented at an uncued location (Klein \& Lawrence, 2012; Theeuwes \& Belopolsky, 2010). When spatial attention is facilitated by the cue, this comparison yields evidence that responding to a cued target location is speeded relative to an uncued location. With respect to eye movements, because they are intimately linked to spatial attention, the shifting of attention to the target location by the cue would speed eye movement initiation to the target (e.g., Adler et al., 2002). To assess the role of bottom-up attention in vaginally and caesarean-section-delivered infants' eye movement responding, a $2 \times 2$ analysis of variance (ANOVA) was conducted on infants' mean pooled latencies, with Cue Condition (cue-target-only and no-cue-target-only) and Birth Experience (vaginal delivery and caesarean-section delivery) as between-subjects factors. This analysis revealed that the main effect of cue condition was significant, $F(1$, $381)=6.17, p<.02$, Cohen's $f=0.12$, indicating that saccade latencies to a cued target $(M=745.82 \mathrm{~ms}, 95 \%$ CI [688.61, 803.02]) were significantly faster than saccade latencies to an uncued target $(M=845.63 \mathrm{~ms}, 95 \%$ CI [781.72, 909.53]). This result demonstrates, consistent with previous spatial-cueing studies (Klein \& Lawrence, 2012; Theeuwes \& Belopolsky, 2010), that the cue facilitated the allocation of spatial attention prior to presentation of the target, thereby speeding the eye movement response to the target.
The main effect of birth experience, $F(1,381)=8.91, p<$ .01 , Cohen's $f=0.14$, was also significant, indicating that the saccade latencies of infants delivered vaginally were faster $(M=762.76 \mathrm{~ms}, 95 \%$ CI $[677.72,787.8])$ than the saccade latencies of infants delivered by caesarean section $(M=855.67 \mathrm{~ms}, 95 \%$ CI $[789.61,921.72])$. As can be seen in Fig. 3, the interaction between cue condition and birth experience was not significant, $F(1,381)=0.37$, n.s., indicating that the saccade latencies of vaginally delivered infants relative to infants delivered by caesarean section were faster whether the target was cued (vaginal, $M=694.27 \mathrm{~ms}, 95 \%$ CI [621.65, 766.89]; caesarean section, $M=796.88 \mathrm{~ms}, 95 \%$ CI $[708.51,885.25]$ ) or was not cued (vaginal, $M=775.31 \mathrm{~ms}$, $95 \%$ CI [691.19, 859.42]; caesarean section, $M=930.19$, $95 \%$ CI [833.64, 1,026.74]). These results demonstrated that, irrespective of whether or not spatial attention was cued, infants who were delivered by caesarean section took longer to allocate attention and initiate that eye movement than those who were delivered vaginally. Consequently, the lack of an interaction suggests that the significantly slower initiation of saccadic eye movements by infants delivered by caesarean section was not a consequence of a unique difficulty in processing the brief cue. Instead, the slower eye movements were likely due to differences between caesarean-section and vaginally delivered infants' mechanisms for initially allocating spatial attention.

To further assess the effect of birth experience on infants' bottom-up spatial attention, we analyzed infants' latencies to initiate saccadic eye movements to a visual target's location as specified by a prior spatial cue, when there was competition for attention relative when there was not. A $2 \times 2$ ANOVA was

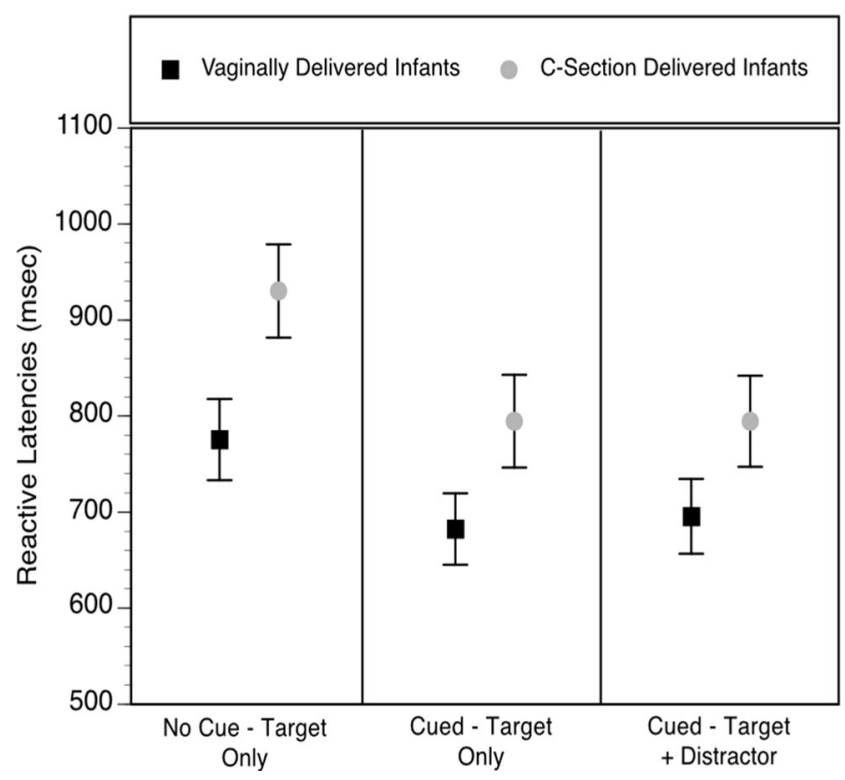

Fig. 3 Mean saccadic latencies in the spatial-cueing task. The results show infants' mean saccadic latencies as a function of whether they were delivered vaginally or by caesarean section for the three spatial-cueing conditions. Error bars indicate $\pm S E S$ 
conducted on infants' mean pooled latencies, with Target Condition (target-only and target + distractor) and Birth Experience (vaginal delivery and caesarean-section delivery) as between-subjects factors. This analysis revealed that the main effect of birth experience, $F(1,352)=6.88, p<.01$, Cohen's $f=$ 0.13 , was significant, indicating that the saccade latencies of infants delivered vaginally were faster $(M=688.64 \mathrm{~ms}, 95 \%$ CI $[635.75,741.53])$ than the saccade latencies of infants delivered by caesarean section $(M=802.25 \mathrm{~ms}, 95 \%$ CI [735.89, 868.6]). The main effect of target condition was not significant, $F(1,352)=0.01$, n.s., indicating that saccade latencies to a target presented alone $(M=744.48 \mathrm{~ms}, 95 \%$ CI [683.88, 805.09]) did not differ significantly from saccade latencies to a target presented with a distractor $(M=746.47 \mathrm{~ms}, 95 \%$ CI [685.97, 806.97]). As can be seen in Fig. 3, the interaction of target condition and birth experience was also not significant, $F(1,352)=0.08$, n.s., indicating that the differences in saccade latencies of vaginally delivered infants relative to infants delivered by caesarean section were similar when a target was presented alone (vaginal, $M=682.42 \mathrm{~ms}, 95 \%$ CI [608.44, 756.4]; caesarean section, $M=807.91 \mathrm{~ms}, 95 \%$ CI $[711.78,904.04])$ and when a target was presented with a distractor (vaginal, $M=$ $695.45 \mathrm{~ms}, 95 \%$ CI [618.28, 772.61]; caesarean section, $M=$ $794.53,95 \%$ CI [700.32, 888.75]). Thus, irrespective of whether infants had to initiate a saccadic eye movement to a single item or to a stimulus item in the presence of a competing item, those who were delivered by caesarean section took longer to allocate attention and initiate that eye movement than did those who were delivered vaginally.

Alternatively, the slowed attentional responses of caesarean-section infants relative to vaginal infants might have been due to other factors. Often, for example, when speeded responses are made, there is a speed-accuracy trade-off in which higher accuracy leads to slower responses, whereas faster responses lead to lower accuracy (Ho et al., 2012; Pachella, Smith, \& Stanovich, 1978). Consequently, both groups of infants' accuracies in making a saccade to the cued target in the target + distractor condition were computed. Contrary to a speed-accuracy trade-off explanation, caesarean-section-delivered infants made errors $(24.18 \%$, $95 \% \mathrm{CI}[19.35,29.00])$ at essentially the same rate as vaginally delivered infants $(29.45 \%, 95 \%$ CI $[22.64,36.26])$, $t(6)=2.01$, n.s., indicating that the caesarean-section infants were not slowing the initiation of their saccadic eye movements in order to more accurately select the cued target. Because of the relatively small sample size, additional data would be required to statistically confirm this finding.

An additional factor that could potentially influence differences between vaginally delivered and caesarean-section infants might be maternal age. Increased maternal age has previously been shown to be a factor in a number of adverse and atypical effects (Koyama, Kamio, Inada, \& Inokuchi, 2011; Menezes et al., 2010; Sandin et al., 2012). Furthermore, increased maternal age is also correlated with an increased likelihood of delivering by caesarean section (Bayrampour \& Heaman, 2010). Consequently, caesarean-section infants' slowed attentional responding might have been due to influences of maternal age rather than to consequences of their birth experience. To assess this possibility, a $3 \times 2$ ANOVA was conducted comparing the mean maternal ages in each of three spatial-cue and no-cue conditions as a function of whether an infant's birth experience was a caesarean section or a vaginal delivery. The results indicated no significant main effect of birth experience, $F(1,14)=0.04$, n.s., nor was the main effect of cueing condition significant, $F(2,14)=0.45$, n.s. These results indicate that maternal age was not different for the caesarean-section infants $(M=33.8$ years, $95 \% \mathrm{CI}$ $[30.00,37.60])$ than for vaginally delivered infants $(M=$ 34.1 years, $95 \%$ CI $[30.81,37.39])$, nor was maternal age different for the different spatial-cueing conditions. The interaction between birth experience and cueing conditions was also not significant, $F(2,14)=0.16$, n.s., indicating that maternal age was the same for the caesarean-section and vaginally delivered infants in each cueing condition. These findings support the interpretation that caesarean-section infants' slower initiation of saccadic eye movements relative to vaginally delivered infants was not due to complications of increased maternal age. Instead, considering that providing a cue speeded eye movements relative to when there was no cue (see Fig. 3) for infants delivered either by caesarean section or vaginally, this suggests that birth experience likely had an impact on the development of the brain area involved in bottom-up, stimulus-driven spatial attention. To reliably implicate bottom-up spatial attention mechanisms, however, would likely require the inclusion of additional conditions such as invalid cues. Furthermore, though the results of this experiment suggest a connection between birth experience and attention and eye movements, because birth experience is not a variable that can be manipulated, a causal relation with attentional responding cannot be assumed.

\section{Experiment 2: Visual expectation task}

\section{Method}

Participants The data from 12 infants ( 7 males, 5 females), who ranged in age from 98 to 125 days old $(M=112.8, S D=$ $11)$, were analyzed in this experiment. The infants were Caucasian $(n=7)$, Asian $(n=4)$, and African $(n=1)$ and were primarily of middle to upper SES. The infants were all born full-term (six delivered via caesarean, six delivered vaginally), in good health, with no apparent visual or neurological abnormalities. The data from 6 additional infants who participated were excluded from the study because of fussiness $(n=4)$ or inattentiveness (i.e., infants were disinterested or looked away 
from the visual field on more than $35 \%$ of trials; $n=2$ ). The sample size of infants for this task was set to be consistent with previous infant visual expectation studies (Adler \& Haith, 2003; Adler et al., 2008; Haith et al., 1988).

Stimuli and apparatus The apparatus, computers, and eyetracking equipment and setup were identical to those aspects of Experiment 1. Infants viewed stimuli in a sequence that was a variation of the visual expectation paradigm (Adler \& Haith, 2003; Haith et al., 1988). The stimuli were computergenerated graphic images of checkerboards, vertical stripes, concentric circles, and diamond-in-square shapes in various combinations of green, red, yellow, and blue (Adler \& Haith, 2003). The infant viewed the images at a distance of $40 \mathrm{~cm}$. The stimuli were approximately $4.5^{\circ}$ squares, and their centers were $5.5^{\circ}$ to the left or right of the infant's visual center. An ISI of 1,000 ms preceded each picture, during which the infants had the opportunity to make anticipatory eye movements in the absence of any visual stimuli (i.e., the monitor screen was blank). A total of 100 pictures were presented to each infant, with the first 10 constituting a baseline period during which the pictures were randomly presented on the two sides and infants' eye movement activity prior to learning was assessed. The remaining 90 pictures constituted the experimental phase, during which every picture on one side of the video monitor appeared in the same color combination (red/green, red/blue, yellow/green, or blue/yellow), whereas the color combination for the pictures on the other side of the video monitor varied randomly among the four color combinations.

Procedure Initially, infants saw 10 images that were presented in an irregular spatial sequence and had random color content, constituting a baseline phase during which infants' saccadic latency and anticipation levels were assessed before expectations were formed. These initial ten baseline trials were included to expose the infants to the locations and type of stimuli used in the experiment. The remaining images served as the experimental phase, during which the pictures were presented in a predictable, alternating left-right sequence and in which the color combination of the stimulus patterns appearing on one side was invariant (e.g., always red/green), whereas the color combination of the stimulus patterns appearing on the the other side varied (e.g., red/green, then blue/yellow, then red/blue, etc.). A schematic of this task is exhibited in Fig. 2B. Each stimulus appeared for a duration of $700 \mathrm{~ms}$, with an ISI of 1,000 ms between the images. Checkerboards, vertical stripes, concentric circles, and diamondshaped stimuli were used in four possible color combinations (red/green, red/blue, yellow/green, and blue/yellow).

Data reduction and analysis As in Experiment 1, the raw digital data recorded by the eyetracker were imported into a MATLAB toolbox called ILAB for analysis (Gitelman, 2002).
Furthermore, the criteria for determining valid and useable eye movements were identical to those used in Experiment 1, with one exception. In the present experiment, only the data of infants $(n=12)$ who attended (looked at the stimuli) on a minimum of $60 \%$ of the trials, instead of $50 \%$ as in Experiment 1, were included (Adler \& Haith, 2003; Adler \& Orprecio, 2006). The minimum attention criterion was set higher for the expectation experiment in order to assure that the infants included in the data analysis had enough exposure to the stimuli and were given enough time to acquire important predictable information about the stimuli.

Infants' eye movements were qualified as belonging to one of two measurement categories that have been used to reflect what we have referred to as anticipation and reactive eye movements (Adler \& Haith, 2003; Haith et al., 1988). Anticipation refers to an appropriate eye movement that is triggered prior to a visual event, whereas reactive refers to the latency of an eye movement following the event that reacts to the onset of that stimulus event. An eye movement was categorized as an anticipation if it occurred during the ISI preceding an event, or within $167 \mathrm{~ms}$ following stimulus onset (i.e., faster than the lower limit of infant reaction time [RT]), and if the movement was directionally appropriate. A percentage-of-anticipation measure was computed by the formula

Number of Anticipation Trials

Number of Anticipation Trials + Number of RT Trials

with the denominator reflecting the total number of pictures on which the scorer judged the infant to be looking. For those occasions on which the infant did not anticipate the event but did make a directionally appropriate eye movement at $168 \mathrm{~ms}$ or later, a latency was recorded.

\section{Results}

Caesarean-section infants' bottom-up, stimulus-driven spatial attention and initiation of saccadic eye movements were shown to be slowed relative to vaginally delivered infants' in the spatial-cueing task. The allocation of spatial attention, however, has been shown to be driven not only in a bottom-up fashion by stimulus properties, but also in a top-down manner by cognitive goals (Theeuwes, 2010; Wolfe \& Horowitz, 2004). Research with infants has shown that they will initiate anticipatory eye movements based on their top-down cognitive expectation of a forthcoming stimulus event (Adler \& Haith, 2003; Adler et al., 2008). To examine whether infants' top-down, goal-driven attention is affected by birth experience, as was their bottom-up attention, their level of anticipatory eye movements was assessed in a visual expectation task in which the stimuli spatially alternated and the stimuli on one side all had an invariant color combination (e.g., red/green), whereas the stimuli on the other side had varied color 
combinations. A $2 \times 2$ ANOVA produced a main effect of color side that was significant, $F(1,20)=19.69, p<.001$, Cohen's $f=0.91$, indicating that, consistent with previous studies (Adler \& Haith, 2003; Wentworth \& Haith, 1992), infants made more anticipatory eye movements to the invariant color side $(M=38.74 \%, 95 \% \mathrm{CI}[35.08,42.4])$ than to the varied color side $(M=28.13 \%, 95 \%$ CI $[24.61,31.66])$. Interestingly, neither the main effect of birth experience, $F(1$, $20)=0.12$, n.s., nor the interaction of birth experience and color side, $F(1,20)=0.40$, n.s., was significant. ${ }^{1}$ As can be seen in Fig. 4, we found no difference between caesareansection and vaginally delivered infants for either the overall level of anticipatory eye movements or the greater number of anticipatory eye movements to the invariant color side. This finding contrasts with the finding of a difference between caesarean-section and vaginally delivered infants in their initiation of bottom-up-guided eye movements in the spatialcueing task.

Infants only initiated top-down anticipatory eye movements on approximately $35 \%$ of the trials; on the rest of the trials, they initiated bottom-up-guided eye movements driven by reaction to the spatial onset of the stimulus. As in the spatial-cueing task, analysis of the latencies of these eye movements revealed that the main effect of color side was not significant, $F(1,20)=0.08$, n.s., indicating that the latencies of reactive eye movements did not differ between the invariant and varied color sides, consistent with previous studies. The main effect of birth experience, as can be seen in Fig. 5, was significant, $F(1,20)=9.41, p<.007$, Cohen's $f$ $=0.62$, indicating that caesarean-section infants were slower to initiate their bottom-up-guided eye movements ( $M=$ $404.92 \mathrm{~ms}, 95 \%$ CI $[373.93,435.91])$ than were vaginally delivered infants ( $M=351.38 \mathrm{~ms}, 95 \%$ CI $[331.65,371.1])$. This finding from a different task provides converging evidence of the attentional responding difference between caesarean-section and vaginally delivered infants found in the spatial-cueing task.

As with the spatial-cueing task, to assess the potential influence of maternal age on the latency of infants' bottom-up reactive saccades as a function of birth experience in the expectation task, maternal age was analyzed as a function of whether infants' birth experience was a caesarean section or a vaginal delivery. This analysis indicated that the maternal ages were not different for caesarean-section infants $(M=31.8$

\footnotetext{
${ }^{1}$ Because one needs to be cautious when interpreting null effects, the lack of a significant difference in anticipatory eye movements between infants delivered by caesarean section and those delivered vaginally could have been due to insufficient power. Consequently, this experiment will need to be replicated with a larger sample. However, if one considers the relatively large effect size from the difference in reactive latencies between infants delivered by caesarean section and those delivered vaginally, we would argue that there was more than enough power to detect any robust effect.
}

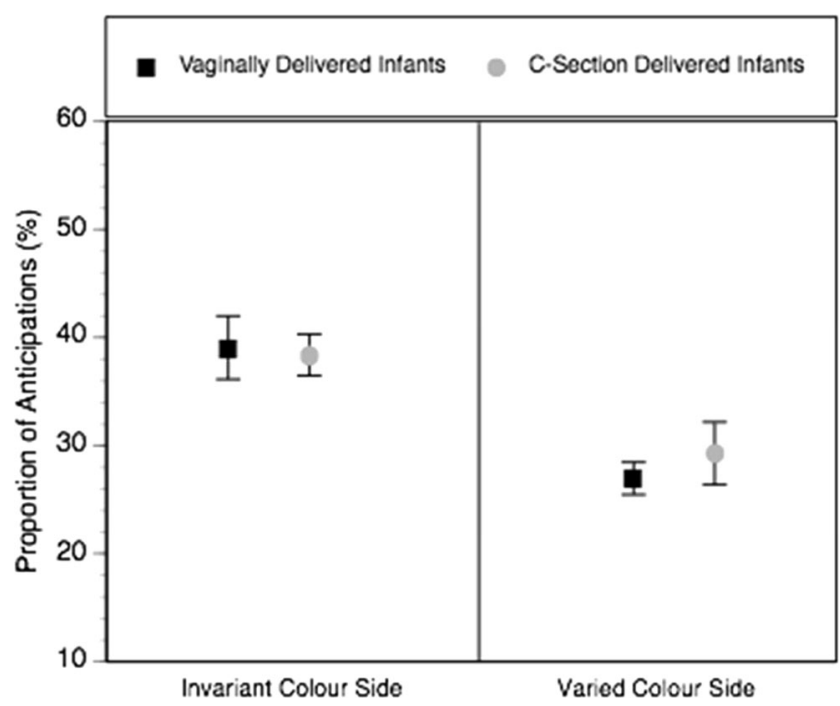

Fig. 4 Frequency of anticipatory saccades in the visual expectation task. The results show infants' percentages of trials on which they made an anticipatory saccadic eye movement to the invariant and varied color combination sides, as a function of whether they were delivered vaginally or by caesarean section. Error bars indicate $\pm S E \mathrm{~s}$

years, $95 \% \mathrm{CI}[373.93,435.91])$ relative to vaginally delivered infants $(M=30.5$ years, $95 \%$ CI $[373.93,435.91]), t(9)=$ 0.44 , n.s. Thus, the slower initiation of reactive saccades in the visual expectation task for infants delivered by caesarean section was not likely due to complications from greater maternal age, relative to infants delivered vaginally.

\section{Discussion}

The event of birth is a major experience in the life of any organism, including humans, yet the consequences of this

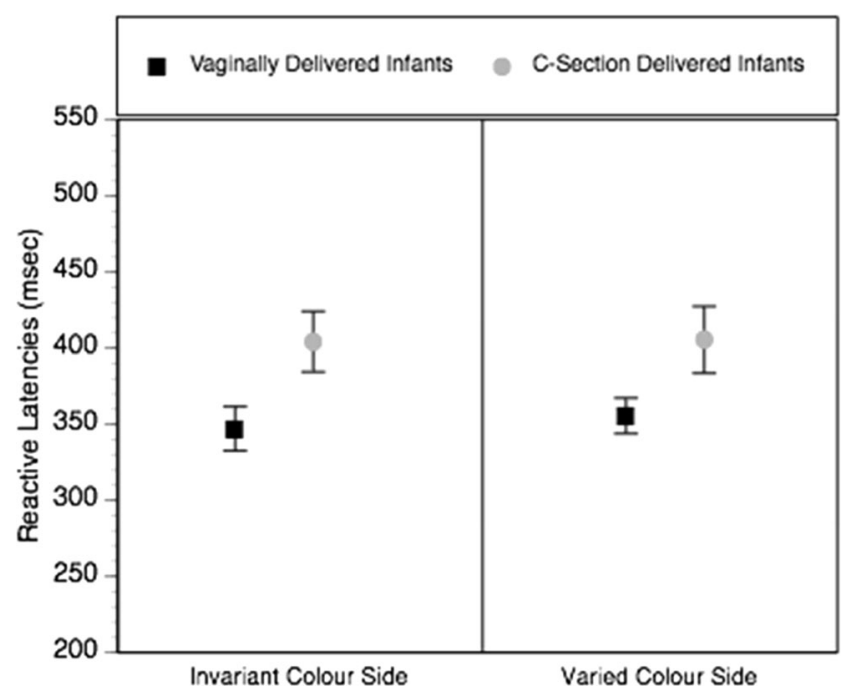

Fig. 5 Mean latencies of reactive saccades in the visual expectation task. The results show infants' mean saccadic latencies to the invariant and varied color combination sides, as a function of whether they were delivered vaginally or by caesarean section. Error bars indicate $\pm S E \mathrm{~s}$ 
experience on any aspect of cognitive and brain development have not been previously reported. In the present study, infants who experienced a caesarean-section birth exhibited slower initiation of saccades driven by bottom-up visual stimulus spatial properties than did infants who experienced a vaginal birth. Yet birth experience had no reliable impact on the initiation of anticipatory saccades driven by top-down cognitive expectations. These results suggest that the type of birth that one experiences interacts with bottom-up, stimulus-driven, but not top-down, cognitively driven spatial attention, though the exact nature and direction of that relation needs to be determined. Given the prior findings of others on the relation between spatial attention and the somatosensory cortex in development (Gilmore et al., 2012; Watanabe, Homae, Nakano, $\&$ Taga, 2008) and the probable impact of birth experience on the development of the somatosensory cortex (Toda et al., 2013), it is possible that one manner by which birth experience might interact with spatial attention reflects the involvement of the somatosensory cortex and its differential development.

Differential attentional responding due to birth experience has a number of implications. The notion that the relation of birth experience to attentional responding is potentially mediated by differences in brain connectivity and the brain's initial state suggests that birth experience should be considered in any investigation and descriptions of brain and cognitive development. Whether the relation between birth experience and attentional responding is a transient effect or is exhibited over the long term is an open question. If the relation exhibited in the present study is transient, then the present study suggests that, at the very least, previous investigations of brain and cognitive development in early infancy may have been modulated by the relative influence of different birth experiences. If the relation is not transient, however, the manner in which birth experience, particularly caesarean section, modulates brain and cognitive development across the lifespan should be investigated.

These investigations will also have to consider whether there is any relation of the effect of birth experience on attentional responding, and possibly brain development, with later exhibition of developmental disabilities, especially since attentional deficits are frequently manifested by members of these groups. Studies using a spatial-cueing task, for example, have demonstrated that individuals with autistic spectrum disorder, as well as children with ADHD, perform more poorly on this attention task than do typical individuals. Landry and Bryson (2004), for example, found that autistics were significantly slower to disengage attention from a fixation location to shift to a cued location than were typical individuals, or even those with Down's syndrome. Similarly, recent research has shown that children with attention-deficit/hyperactivity disorder exhibit slowed RTs to a cued target (Ortega, López, Carrasco, Anllo-Vento, \& Aboitiz, 2013). Whether there is a connection between birth experience, particularly caesarean- section delivery, and the attentional deficits seen in some developmental disabilities is clearly speculative, but an intriguing question nonetheless.

Although maternal age was assessed relative to whether infants were born by caesarean section or vaginally and was found not to differ, additional factors other than differences in brain development could have contributed to the poorer attentional responding in caesarean-section infants. Some of the other factors that might distinguish infants born by caesarean section from those born vaginally include, but are not limited to, maternal weight (Chu et al., 2007; Galtier-Dereure, Montpeyroux, Boulot, Bringer, \& Jaffiol, 1995), fetal status and weight (Barber et al., 2011), and position of the fetus in the uterus (Akmal, Kametas, Tsoi, Howard, \& Nicolaides, 2004). Each of these factors has been associated with an increased probability of caesarean-section delivery, and consequently, each factor might contribute to the attentional deficit of caesarean-section delivery revealed in the present study. Other factors that will need to be considered include the infant's gestational age, Apgar score, and weight, as well as the method of feeding (breast or bottle feeding) that the mother uses. Furthermore, caesarean sections can be categorized into two types: planned, in which case the mother and infant do not experience any labor, and unplanned, in which case the mother and infant might experience some labor. Whether the different experiences of these two types of caesarean section differentially influence attentional responding due to disparate effects on brain development is an open question. Future studies will need to titrate the relative influences of each of these and other factors on the apparently slower attentional responding of infants delivered by caesarean section.

The importance of understanding the possible brain connectivity and cognitive impacts of caesarean-section delivery is amplified with respect to both research and health implications, because the number of caesarean sections being performed has been steadily increasing (Martin, Hamilton, Ventura, Osterman, \& Mathews, 2013). In fact, others have sounded the alarm in terms of the medical implications of an increasing caesarean-section delivery rate (American College of Obstetricians \& Gynecologists et al., 2014) and have expressed caution at its continuing increased usage and health impact. In the present study, a potential psychological implication has been added to the roster of impacts of caesareansection delivery.

Author note This research was supported by Grant Number R03MH085994-01A1 from the National Institute of Mental Health and by a York University Faculty of Health Minor Research Grant awarded to the first author. An earlier version of these data was presented at the meeting of the Canadian Society for Brain, Behavior, and Cognitive Science in Toronto in July 2014. 


\section{References}

Adler, S. A., \& Gallego, P. (2014). Search asymmetry and eye movements in infants and adults. Attention, Perception, and Psychophysics, 76, 1590-1608. doi:10.3758/s13414-014-0667-6

Adler, S. A., \& Haith, M. M. (2003). The nature of infants' visual expectations for event content. Infancy, 4, 389-421.

Adler, S. A., \& Orprecio, J. (2006). The eyes have it: Visual pop-out in infants and adults. Developmental Science, 9, 189-206.

Adler, S. A., Bala, J., \& Krauzlis, R. J. (2002). Primacy of spatial information in guiding target selection for pursuit and saccades. Journal of Vision, 2, 627-644. doi:10.1167/2.9.5

Adler, S. A., Haith, M. M., Arehart, D. M., \& Lanthier, E. C. (2008). Infants' visual expectations and the processing of time. Journal of Cognition and Development, 9, 1-25.

Akmal, S., Kametas, N., Tsoi, E., Howard, R., \& Nicolaides, K. H. (2004). Ultrasonographic occiput position in early labour in the prediction of caesarean section. BJOG: An International Journal of Obstetrics and Gynecology, 111, 532-536.

American College of Obstetricians and Gynecologists, Society for Maternal-Fetal Medicine, Caughey, A. B., Cahill, A. G., Guise, J.M., \& Rouse, D. J. (2014). Safe prevention of the primary cesarean delivery. American Journal of Obstetrics and Gynecology, 210, 179-193.

Aoki, C., \& Erisir, A. (2013). Experience-dependent synaptic plasticity in the developing cerebral cortex. In V. Pickel \& M. Segal (Eds.), The synapse: Structure and function (pp. 397-420). Oxford: Elsevier.

Atkinson, J. (1984). Human visual development over the first 6 months of life. A review and a hypothesis. Human Neurobiology, 3, 61-74.

Atkinson, J. (2000). The developing visual brain. Oxford: Oxford University Press.

Balslev, D., Odoj, B., \& Karnath, H. (2013). Role of somatosenosry cortex in visuospatial attention. Journal of Neuroscience, 33, 18311-18318.

Barber, E. L., Lundsberg, L., Belanger, K., Pettker, C. M., Funai, E. F., \& Illuzzi, J. L. (2011). Obstetrics and Gynecology, 118, 29-38.

Bayrampour, H., \& Heaman, M. (2010). Advanced maternal age and the risk of cesarean birth: A systematic review. Birth, 37, 219-226.

Braddick, O., \& Atkinson, J. (2011). Development of human visual function. Vision Research, 51, 1588-1609.

Canfield, R. L., Smith, E. G., Brezsnyak, M. P., \& Snow, K. L. (1997). Information processing through the first year of life: A longitudinal study using the visual expectation paradigm. Monographs of the Society for Research in Child Development, 62(2), 1-145.

Cardwell, C. R., Stene, L. C., Stoner, G., Cinek, O., Svensson, J., Goldacre, M. J., . . . Patterson, C. C. (2008). Caesarean section is associated with an increased risk of childhood-onset type 1 diabetes mellitus: A meta-analysis of observational studies. Diabetologia, 51, 726-735.

Chu, S. Y., Kim, S. Y., Schmid, C. H., Dietz, P. M., Callaghan, W. M., Lau, J., \& Curtis, K. M. (2007). Maternal obesity and risk of cesarean delivery: A meta-analysis. Obesity Reviews, 8, 385-394.

Diamond, A., \& Amso, D. (2008). Contributions of neuroscience to our understanding of cognitive development. Current Directions of Neuroscience, 17, 136-141. doi:10.1111/j.1467-8721.2008.00563.x

Flemming, K., Woolcott, C. G., Allen, A. C., Veugelers, P. J., \& Kuhle, S. (2013). The association between caesarean section and childhood obesity revisited: A cohort study. Archives of Disease in Childhood, 98, 526-532.

Galtier-Dereure, F., Montpeyroux, F., Boulot, P., Bringer, J., \& Jaffiol, C. (1995). Weight excess before pregnancy: Complications and cost. International Journal of Obesity and Related Metabolic Disorders, 19, 443-448.

Gilmore, R. O., \& Johnson, M. H. (1995). Working memory in infancy: Six-month-olds' performance on two versions of the oculomotor delayed response task. Journal of Experimental Child Psychology, 59, 397-418.

Gilmore, J. H., Shi, F., Woolson, S. L., Knickmeyer, R. C., Short, S. J., Lin, W., . . Shen, O. (2012). Longitudinal development of cortical and subcortical gray matter from birth to 2 years. Cerebral Cortex, $22,2478-2485$.

Gitelman, D. R. (2002). ILAB: A program for post experimental eye movement analysis. Behavior Research Methods, Instruments, \& Computers, 34, 605-612. doi:10.3758/BF03195488

Greenough, W. T., \& Black, J. E. (2013). Induction of brain structure by experience: Substrates for cognitive development. In M. R. Gunnar \& C. A. Nelson (Eds.), Developmental behavioral neuroscience: The Minnesota Symposia on Child Psychology (Vol. 24, pp. 155200). New York: Psychology Press.

Haith, M. M., Hazan, C., \& Goodman, G. S. (1988). Expectation and anticipation of dynamic visual events by 3.5 -month-old babies. Child Development, 59, 467-479.

Ho, T., Brown, S., van Maanen, L., Forstmann, B. U., Wagenmakers, E.J., \& Serences, J. T. (2012). The optimality of sensory processing during speed-accuracy tradeoff. Journal of Neuroscience, 32, 79928003. doi:10.1523/JNEUROSCI.0340-12.2012

Hoffman, J. E., \& Subramaniam, B. (1995). The role of visual attention in saccadic eye movements. Perception \& Psychophysics, 57, 787795. doi:10.3758/BF03206794

Hunnius, S., \& Geuze, R. H. (2004). Gaze shifting in infancy: A longitudinal study using dynamic faces and abstract stimuli. Infant Behavior and Development, 27, 397-416.

Johnson, M. H. (1995). The development of visual attention: A cognitive neuroscience perspective. In M. S. Gazzinga (Ed.), The cognitive neurosciences (pp. 735-747). Cambridge: MIT Press.

Johnson, M. H. (2002). The development of visual attention: A cognitive neuroscience perspective. In M. H. Johnson, Y. Munakata, \& R. Gilmore (Eds.), Brain development and cognition: A reader (pp. 134-150). Oxford: Blackwell Press.

Jones, S. R., Kerr, C. E., Wan, Q., Pritchett, D. L., Hämäläinen, M., \& Moore, C. I. (2010). Cued spatial attention drives functionally relevant modulation of the mu rhythm in primary somatosensory cortex. Journal of Neuroscience, 30, 13760-13765. doi:10.1523/ JNEUROSCI.2969-10.2010

Klein, R. M., \& Lawrence, M. A. (2012). On the modes and domains of attention. In M. I. Posner (Ed.), Cognitive neuroscience of attention (pp. 11-48). New York: Guilford Press.

Kowler, E., Anderson, E., Dosher, B., \& Blaser, E. (1995). The role of attention in the programming of saccades. Vision Research, 35, 1897-1916. doi:10.1016/0042-6989(94)00279-U

Koyama, T., Kamio, Y., Inada, N., \& Inokuchi, E. (2011). Maternal age at childbirth and social development in infancy. Research in Autism Spectrum Disorders, 5, 450-454.

Landry, R., \& Bryson, S. E. (2004). Impaired disengagement of attention in young children with autism. Journal of Child Psychology and Psychiatry, 45, 1115-1122.

Martin, J. A., Hamilton, B. E., Ventura, S. J., Osterman, M. J., \& Mathews, T. J. (2013). Births: Final data for 2011. National Vital Statistics Report, 62, 1-90.

Maurer, D., Ellemberg, D., \& Lewis, T. L. (2006). Repeated measurements of contrast sensitivity reveal limits to visual plasticity after early binocular deprivation in humans. Neuropsychologia, 44, 2104-2112. doi:10.1016/j.neuropsychologia.2005.10.008

Menezes, P. R., Lewis, G., Rasmussen, F., Zammit, S., Sipos, A., Harrison, G. L., . . . Gunnell, D. (2010). Paternal and maternal ages at conception and risk of bipolar affective disorder in their offspring. Psychological Medicine, 40, 477-485.

Mohades, S. G., Struys, E., Van Schuerbeek, P., Mondt, K., Van De Craen, P., \& Luypaert, R. (2012). DTI reveals structural differences in white matter tracts between bilingual and monolingual children. Brain Research, 1435, 72-80. doi:10.1016/j.brainres.2011.12.005 
Ortega, R., López, V., Carrasco, X., Anllo-Vento, L., \& Aboitiz, F. (2013). Exogenous orienting of visual-spatial attention in ADHD children. Brain Research, 1493, 68-79. doi:10.1016/j.brainres. 2012.11.036

Pachella, R. G., Smith, J. K., \& Stanovich, K. E. (1978). Qualitative error analysis and speeded classification. Cognitive Theory, 3, 169-198.

Posner, M. I. (1980). Orienting of attention. Quarterly Journal of Experimental Psychology, 32, 3-25. doi:10.1080/ 00335558008248231

Quigley, M. A., Hockley, C., Carson, C., Kelly, Y., Renfrew, M. J., \& Sacker, A. (2012). Breastfeeding is associated with improved child cognitive development: A population-based cohort study. Journal of Pediatrics, 160, 25-32.

Renz-Polster, H., David, M. R., Buist, A. S., Vollmer, W. M., O’Connor, E. A., Frazier, E. A., \& Wall, M. A. (2005). Caesarean section delivery and the risk of allergic disorders in childhood. Clinical and Experimental Allergy, 35, 1466-1472.

Sandin, S., Hultman, C. M., Kolevzon, A., Gross, R., MacCabe, J. H., \& Reichenberg, A. (2012). Advancing maternal age is associated with increasing risk for autism: A review and meta-analysis. Journal of the American Academy of Child Psychiatry, 51, 477-486.

Sepkoski, C. M., Garcia-Coll, C., \& Lester, B. M. (1982). The cumulative effects of obstetric risk variables on newborn behavior. In L. P. Lipsitt \& T. M. Field (Eds.), Infant behavior and development: Perinatal risk and newborn behavior (pp. 33-39). Norwood: Ablex.

Sepkoski, C. M., Lester, B. M., Ostheimer, G. W., \& Brazelton, T. B. (1992). The effects of maternal epidural anesthesia on neonatal behavior during the first month. Developmental Medicine and Child Neurology, 34, 1072-1080.
Shum, D., Neulinger, K., O’Callaghan, M., \& Mohay, H. (2008). Attentional problems in children born very preterm or with extremely low birth weight at 7-9 years. Archives of Clinical Neuropsychology, 23, 103-112.

Theeuwes, J. (2010). Top-down and bottom-up control of visual selection. Acta Psychologica, 135, 77-99. doi:10.1016/j.actpsy.2010.02. 006

Theeuwes, J., \& Belopolsky, A. (2010). Top-down and bottom-up control of visual selection: Controversies and debate. In V. Coltheart (Ed.), Tutorials in visual cognition (pp. 67-92). New York: Psychology Press.

Toda, T., Homma, D., Tokuoka, H., Hayakawa, I., Sugimoto, Y., Ichinose, H., \& Kawasaki, H. (2013). Birth regulates the initiation of sensory map formation through serotonin signaling. Developmental Cell, 27, 32-46. doi:10.1016/j.devcel.2013.09.002

van de Weijer-Bergsma, E., Wijnroks, L., \& Jongmans, M. J. (2008). Attention development in infants and preschool children born preterm: A review. Infant Behavior and Development, 31, 333-351.

Van der Stigchel, S., \& Theeuwes, J. (2007). The relationship between covert and overt attention in endogenous cueing. Perception \& Psychophysics, 69, 719-731. doi:10.3758/BF03193774

Watanabe, H., Homae, F., Nakano, T., \& Taga, G. (2008). Functional activation in diverse regions of the developing brain of human infants. NeuroImage, 43, 346-357. doi:10.1016/j.neuroimage.2008. 07.014

Wentworth, N., \& Haith, M. M. (1992). Event-specific expectations in 2and 3-month-old infants. Developmental Psychology, 34, 247-257.

Wolfe, J. M., \& Horowitz, T. S. (2004). What attributes guide the deployment of visual attention and how do they do it? Nature Reviews Neuroscience, 5, 495-501. doi:10.1038/nrn1411 